\title{
Aspergeillus Fumigatus: The Effects of Adiponectin on Inflammatory Cytokine Production
}

\author{
Rick Foust and Robert Templeton \\ Indiana University School of Medicine Terre Haute, Department of Microbiology
}

\begin{abstract}
Background and Hypothesis: Although inflammatory cytokines are important for antifungal defenses, excessive production significantly increases host immune pathology. It is therefore important to identify host pathways that limit detrimental inflammation in invasive fungal infection. Our prior results showed that mice with invasive aspergillosis (IA) that were deficient in the metabolic cytokine production produced more of the cytokine Tumor Necrosis Factor (TNF) than alveolar in wild-type control mice. Therefore, we hypothesize that adiponectin inhibits antifungal cytokine secretion in alveolar macrophages.
\end{abstract}

Experimental Design or Project Methods: To test this hypothesis, the commonly used A. fumigatus strain Af293 was purchased from the Fungal Genetics Stock Center and grown on and harvested from agar. The alveolar macrophage cell line MH-S and cytokine ELISA kits was obtained from MilliporeSigma and ThermoFisher, respectively. MH-S cells were stimulated with swollen, fixed Af293 conidia for 24 hours in the presence or absence of recombinant mouse adiponectin. After 24 hours, supernatants and cells was were collected and assayed for ILs 1a, 6 , and TNF protein and mRNA, by ELISA and quantitative RT-PCR, respectively.

Results: Although our preliminary results suggest possible inhibition of cytokine secretion by adiponectin in response to $A$. fumigatus, significant differences have thus far not been observed.

Conclusion and Potential Impact: We are therefore currently optimizing our experimental conditions to improve antifungal cytokine secretion. These studies may ultimately assist in the discovery of novel therapeutic targets and improve the prognosis of $A$. fumigatus infections 